\title{
Numerical Simulation of Internal Flow Field in Solid Rocket Motor with Complex Grain
}

\author{
Yang Yu-long ${ }^{1, a}$, Zheng Jian ${ }^{1, b^{*}}$ \\ ${ }^{1}$ Department of Computing, Main University, Nanjing, China \\ a yangy_long@163.com, b zhengjian@njust.edu.cn \\ *corresponding author:Zheng Jian
}

Keywords: Solid rocket motor, complex grain, numerical simulation

\begin{abstract}
Solid rocket motor is widely used as power device of rocket weapon, and numerical simulation is an important method in the design process of solid rocket motor. A large aspect ratio solid rocket motor with the combination of simple hole grain and aft slot grain was analyzed in this paper. Numerical simulation of the flow field for 4 moments with $k-\varepsilon$ method was carried out to obtain the flow parameters inside the combustion chamber and nozzle. Results shows velocity in the slots is slower than mainstream and vortex exist in the slots and the nozzle, which will strengthen erosive burning of the grain and the ablation in the nozzle.
\end{abstract}

\section{Introduction}

Solid rocket motors are the power equipment for missiles, space vehicles and other aircrafts. Complex grain came into being to meet the require of thrust-time curve in the design process of solid rocket motor.

Lu, Jian-Ying analyzed simulation of impacting ignition of solid rocket motor, and reported that that the critical speed of solid rocket motor firing was related to the mass of solid motor [1]. Liu, Zhong-Bing investigated low temperature ignition adaptability with simulation and experiment, obtained the structure safety margin of each full-scale solid motor [2]. Mərmureanu, Marius Ionut used different burning rate models to simulate internal ballistics in solid rocket motor, obtained determination of different impacts on the actual burning rate, developed 0-D internal ballistic module, which has a high degree of accuracy information on solid rocket motor performances [3]. Javed, A. studied numerical simulation of a dual pulse solid rocket motor with solving three dimensional Reynolds Averaged Navier Stokes equations, captured all the essential features of the flow field [4]. French, Ainslie D. focused on corner and parietal vortex shedding instabilities in computational analysis of the combusted gases passing through the chamber of a solid rocket motor [5]. Chen, Wei carried out the simulation of multi-burning-rate based on parameterized feature CAD 3-D grain regression model, got high accuracy results that provides a practical approach to aid SRM design of multi-burning-rate grain [6]. Li, Jian analyzed a two-dimensional axisymmetric model to predict the ablation of the insulation layer in an end burning rocket motor, results shows that that the erosion rate increase with the increasing of burning time [7].

Four different combustion chamber - nozzle integrated three-dimensional flow field model was established at four moments in this paper. The hypothesis of parallel layer combustion and a $k-\varepsilon$ turbulence model was used with the software Fluent to perform numerical simulations for four moments, to analyze the distribution of parameters such as velocity, pressure and other parameters in the flow field. 


\section{Numerical Simulation}

\subsection{Basic Assumptions}

Problems related to inner flow field are examined in this paper and does not involve complex and integrated aspects such as multiphase flow and combustion. Before making numerical simulations, the following assumptions are made:

(1) The surfaces of the motor shell and nozzle are adiabatic wall.

(2) The deformation of grain under pressure conditions is not considered.

(3) Irrespective of erosion effects.

(4) The combustion is done immediately on the surface of the propellant.

\subsection{The Establishment of Flow Field Model}

A complex grain rocket motor with an aspect ratio of approximately 17 is studied in this paper. According to the analysis of the flow field, the combustion of the grain can be divided into four periods. The first period occurs when the engine starts working until the slots meet. The second period intersects from when the slots meet to the slots touch the engine housing. The third period occurs from the end of the second period until the grain of the slot grain is completely exhausted (burning of the slot residue), and the fourth period is the complete combustion of the remaining propellant. Take the initial time as $t_{1}$, and define the intersecting moments of the four time periods as $t_{2}, t_{3}, t_{4}$,respectively, as shown in Figure 1 to Figure 4.
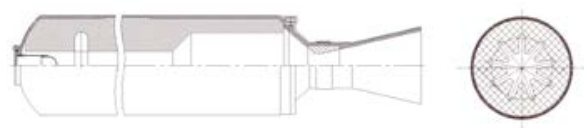

Figure 1 Schematic diagram of grain at $t_{1}$
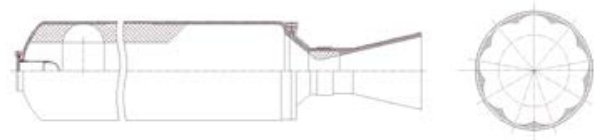

Figure 3 Schematic diagram of grain at $t_{3}$
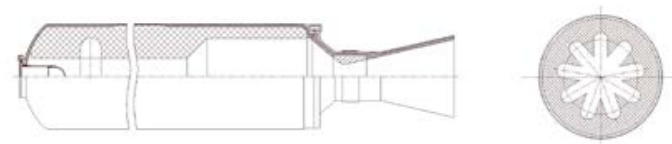

Figure 2 Schematic diagram of grain at $t_{2}$

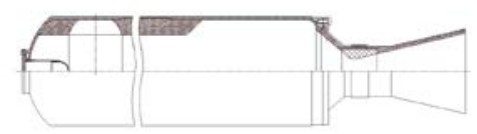

Figure 4 Schematic diagram of grain at $t_{4}$

\subsection{Boundary Conditions}

\section{(1) Mass flow inlet}

The flow rate at the four moments of the flow field vary due to the change of combustion surface and pressure. According to the density of the propellant and the burning rate, the mass flow rate at $\mathrm{t}_{1}$ is $1.464 \mathrm{~kg} / \mathrm{s}$, and the mass flow rate at $t_{2}$ is $1.704 \mathrm{~kg} / \mathrm{s}$, the mass flow rate at $t_{3}$ is $2.499 \mathrm{~kg} / \mathrm{s}$, and the mass flow rate at $t_{4}$ is $2.080 \mathrm{~kg} / \mathrm{s}$.

(2) Pressure outlet

The position of the pressure outlet is the outlet of the nozzles, the pressure is taken as $101325 \mathrm{~Pa}$.

(3) Periodic

Because the flow field is an axisymmetric structure and a total of nine slots existed, one-ninth of the flow field model is studied in this paper for numerical simulation.

(4) Wall

After the above boundary is set, all remaining surfaces are walls. The wall conditions are no slip and a constant temperature of $300 \mathrm{~K}$ for the temperature condition.

\section{Analysis of Simulation Results}

The density-based implicit solver is used in this paper to perform steady-state numerical simulations at four moments. 
Firstly, the longitudinal section of the simulated internal flow field is selected for analysis. Figure 5 to Figure 8 are pressure distributions in longitudinal section from $t_{1}$ to $t_{4}$. It can be seen from the figure that the pressure at the nozzle drops sharply, especially in the throat changes the most. At $t_{1}$ and $t_{2}$ the pressure drops in the combustion chamber and the rate of decline at $t_{1}$ is greater than at $t_{2}$.
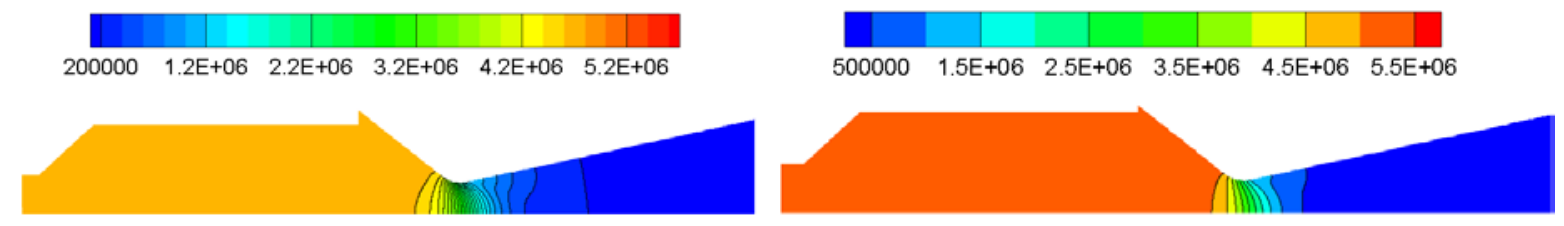

Figure 5 Longitudinal pressure distribution at $t_{1} \quad$ Figure 6 Longitudinal pressure distribution at $t_{2}$
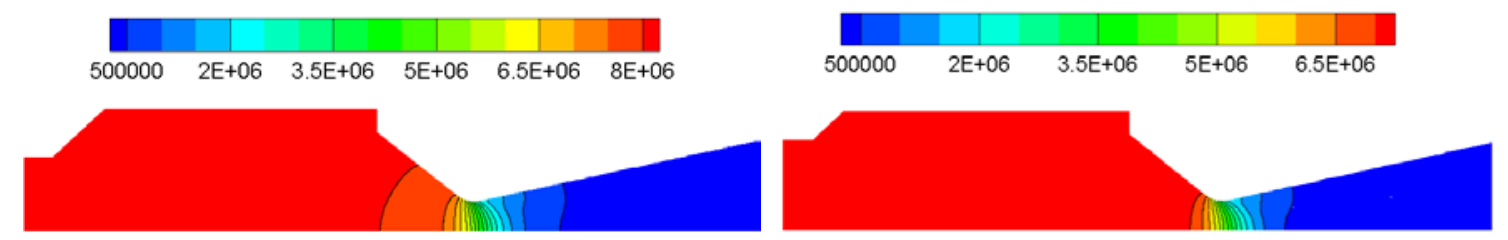

Figure 7 Longitudinal pressure distribution at $t_{3}$ Figure 8 Longitudinal pressure distribution at $t_{4}$

Figure 9 shows velocity distribution at $t_{1}$, the velocity does not change much in the simple hole grain segment, and the flow velocity in the slot is lower than the velocity in the center.

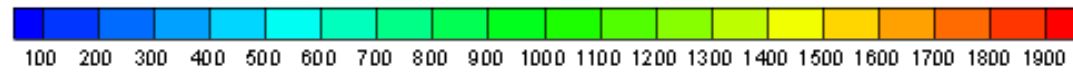

Figure 9 Longitudinal velocity distribution at $t_{1}$

Heat energy contained in the gas and the pressure potential energy are converted into kinetic energy in the procedure of the gas though the nozzle causes acceleration and expansion of the gas. As shown in Figure 10 to Figure 13 ,there are no significant changes in the Mach number in the combustion chamber at $t_{3}$ and $t_{4}$, Mach number in the slot is lower than mainstream Mach number at $t_{1}$ and $t_{2}$.

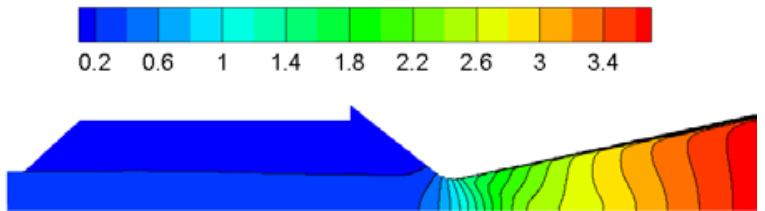

Figure 10 Mach number distribution at $t_{1}$

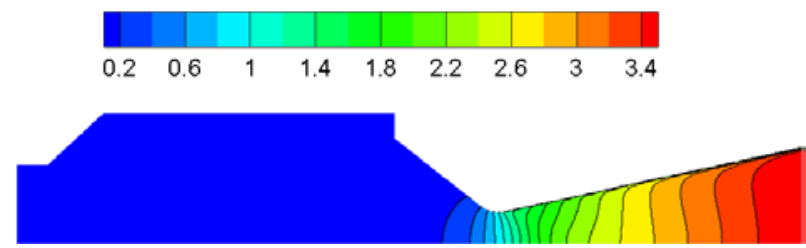

Figure 12 Mach number distribution at $t_{3}$

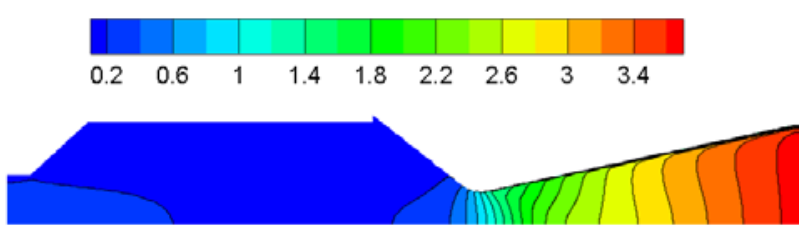

Figure 11 Mach number distribution at $t_{2}$

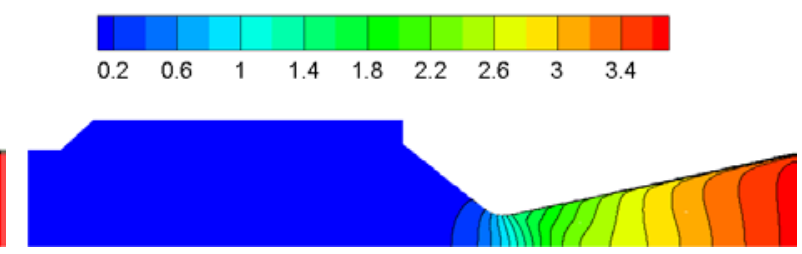

Figure 13 Mach number distribution at $t_{4}$

The results above show that the pressure distribution and Mach number are all distributed smoothly in the combustion chamber and change drastically within the Laval nozzle, especially in the throat, the pressure decrease and the speed increases.

The flow velocity distribution at $t_{1}$ and $t_{2}$ in the slot is as shown lower than the main flow velocity in the Figure14 and Figure 15. The three-dimensional parameter distribution gradually disappears as the three-dimensional model becomes two-dimensional. 


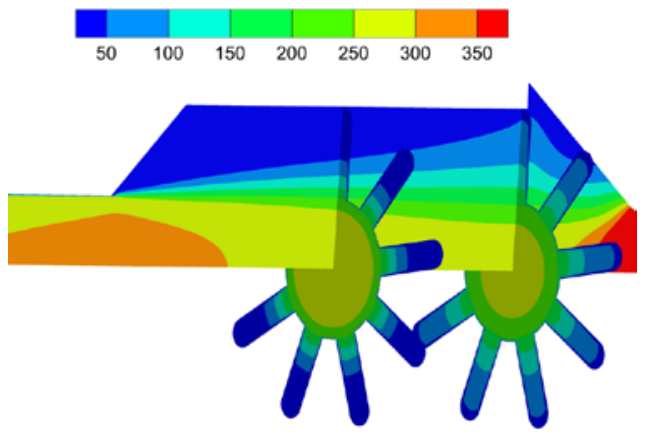

Figure 14 Velocity distribution in the slot at $t_{1}$

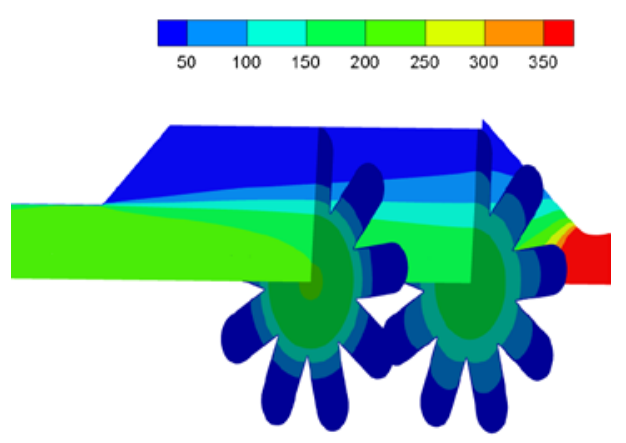

Figure 15 Velocity distribution in the slot at $t_{2}$

Figure 16 and Figure 17 are the distribution of the pressure and velocity along the axis of the flow field different moments. It can be seen that at $t_{1}$ of the simple hole grain section, the burning surface to port area ratio is relatively large, which makes the pressure drops faster compared with other moments. At $t_{2}$, the burning surface has changed and the volume of the flow field has increased, the pressure drop has eased and the velocity has risen slower along the axis than $t_{2}$. At the two moments $t_{3}$ and $t_{4}$, the burning surface to port area ratio decreases with respect to the first two moments, as a result, the decrease in the static pressure and the increase in speed along the axis reduced.

Moreover, the pressure at $t_{1}, t_{2}$ and $t_{3}$ are in the rising state and the velocity distribution are in the decreasing state in the slot grain section and there is little change in the parameters above at $t_{4}$.
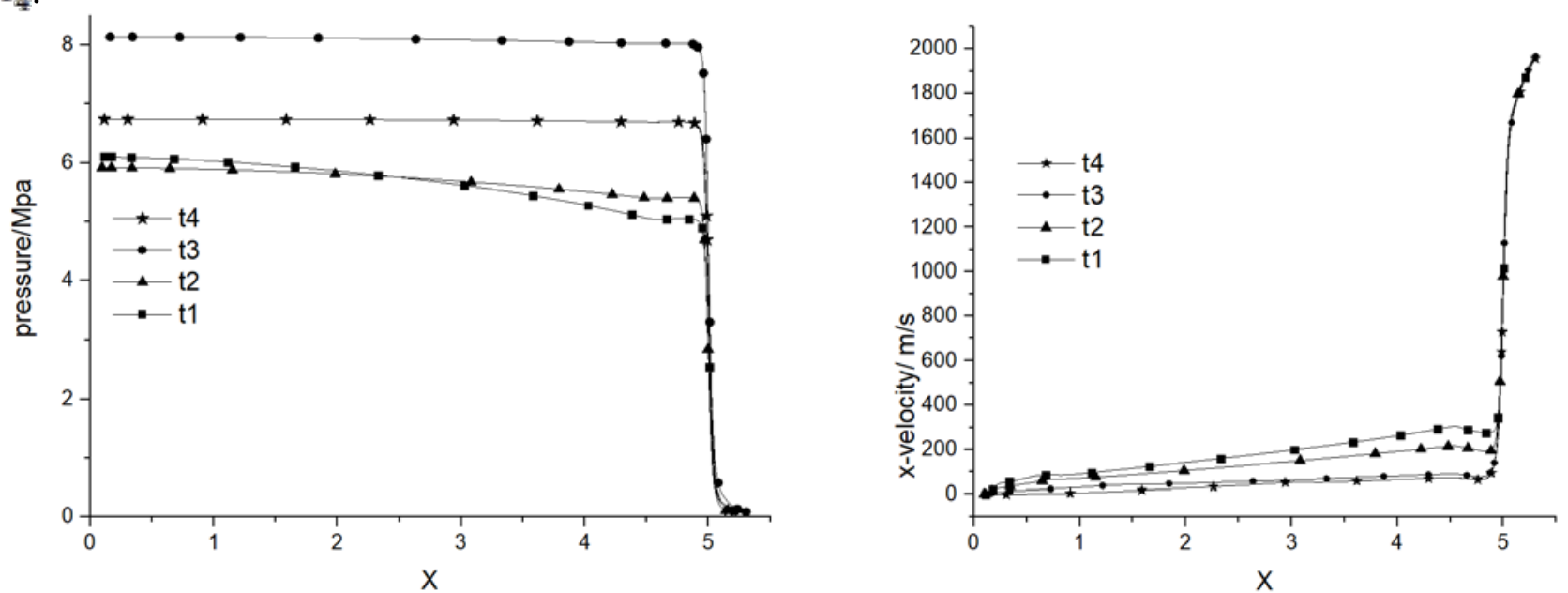

Figure 16 Pressure distribution at flow field axis Figure 17 Velocity distribution at flow field axis

Figure 18 to Figure 20 shows large vortex appears at $t_{1}, t_{2}$, and $t_{3}$ in the slot, due to the sudden expansion of the flow field in slot grain section flow field end face. At $t_{4}$, the mass flow inlet changes into wall, the vortex becomes smaller as is shown in Figure.

As the volume of the flow field increases, the flow pattern in the slot and in the nozzle changes. Vortex exist in the convergence section of the combustion chamber and the nozzle at $t_{1}$ and $t_{2}$ due to the blockage of the grain to the inlet of the nozzle as shown in Figure 22 and Figure 23.

As a result, the influence of the erosive combustion and the thermal protection of the nozzle to prevent ablation caused by the vortex need taken into account since the existence of vortex in the combustion chamber and the nozzle. 


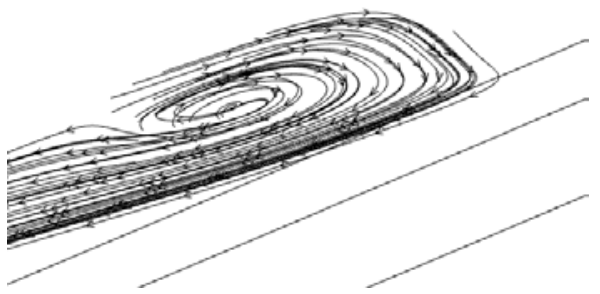

Figure 18 Streamline in the slot at $t_{1}$

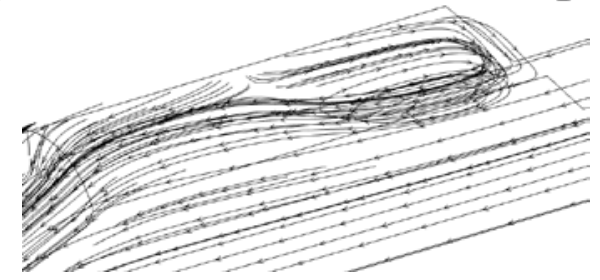

Figure 20 Streamline in the slot at $t_{3}$

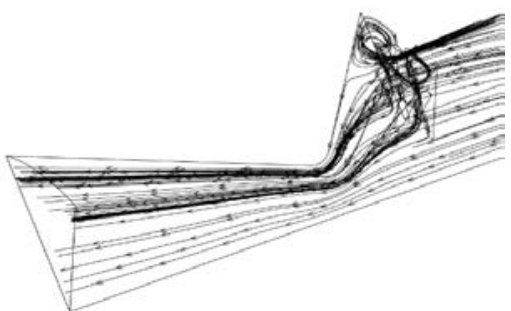

Figure 22 Streamline in the nozzle at $t_{1}$

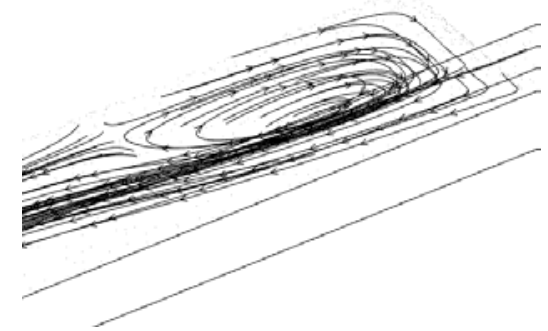

Figure 19 Streamline in the slot at $t_{2}$

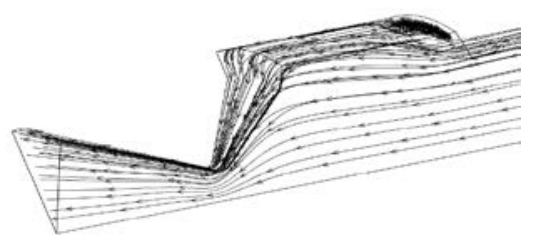

Figure 21 Streamline in the slot at $t_{4}$

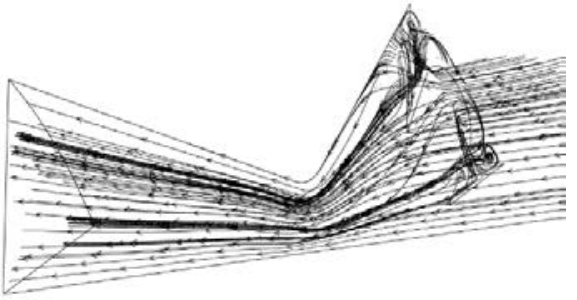

Figure 23 Streamline in the nozzle at $t_{4}$

\section{Conclusion}

Numerical simulation was carried out in this paper to simulate the internal flow of a complex grain solid rocket motor with a combination of simple hole grain and slot grain at four certain moments and analyze the results. The following conclusions are obtained:

In the simple hole grain flow field section, velocity increase and pressure decrease along the axis of the flow field, but due to the influence of the slot, the flow velocity decrease and pressure increase along the axis of the slot grain flow field section. And the flow velocity in the slot is lower than the velocity in the center of the slot grain flow field section. The change of parameters in the combustion chamber such as velocity and pressure along the axis is relatively large at $t_{1}$ compare to other moments, and gradually decrease as time goes on, and drastic changes along the axis occur in the nozzle.

Vortex exist in the slot grain section flow field, which will increase the erosion effect, and the vortex gradually decrease with time goes on. The presence of vortex inside the nozzle will convert the kinetic energy of the gas into heat energy, strengthen the heat transfer to the wall. Strengthen the thermal protection is needed when designing the nozzle.

\section{References}

[1] Lu, Jian-Ying., Chen, Lang., Wang, Chen. and Wu, Jun-Ying. (2013) Numerical simulation of impacting ignition of solid rocket motor, Beijing Ligong Daxue Xuebao/Transaction of Beijing Institute of Technology, 33, 39-43.

[2] Liu, Zhong-Bing., Zhang, Bing. and Zhou, Yan-Qing. (2015) Simulation experiment technology for low temperature ignition adaptability of solid rocket motor, Guti Huojian Jishu/Journal of Solid Rocket Technology, 38, 203-207 and 213.

[3] Mərmureanu, Marius Ionuţ. (2014) Solid rocket motor internal ballistics simulation using different burning rate models, UPB Scientific Bulletin, 76, 49-56.

[4] Javed, A., Manna, P., Chakraborty, Debasis (2012) Numerical simulation of a dual pulse solid 
rocket motor flow field, Defence Science Journal, 62, 369-374.

[5] French, Ainslie D., Panelli, Mario., Di Lorenzo, Giuseppe., Schettino, Antonio., Paglia, Fabio. (2017) Combustion instability and Pressure oscillation numerical simulation in a solid rocket motor, 53rd AIAA/SAE/ASEE Joint Propulsion Conference.

[6] Chen, Wei., Liang, Guo-Zhu. (2015) Internal ballistic simulation of multi-burning-rate solid rocket motor based on parameterized feature CAD model, Hangkong Dongli Xuebao/Journal of Aerospace Power, 30, 3017-3028.

[7] Li, Jian., Zhang, Mei., Zhang, Jiang-Tao. (2014) Numerical simulation of insulation layer ablation in solid rocket motor based on fluent, Applied Mechanics and Materials, 574, 224-229. 